\title{
Bioinformation
}

\section{E-Learning as a new tool in bioinformatics teaching}

\author{
Vijayakumar Saravanan ${ }^{1}$ and Piramanayagam Shanmughavel ${ }^{1, *}$ \\ ${ }^{1}$ DBT Bioinformatics Facility, Department of Bioinformatics, Bharathiar University, Coimbatore 641046, TamilNadu, India; \\ *Piramanayagam Shanmughavel - E-mail: shanvel_99@yahoo.com; ${ }^{*}$ Corresponding author
}

received August 01, 2007; accepted October 16, 2007; published online November 01, 2007

\begin{abstract}
In recent years, virtual learning is growing rapidly. Universities, colleges, and secondary schools are now delivering training and education over the internet. Beside this, resources available over the WWW are huge and understanding the various techniques employed in the field of Bioinformatics is increasingly complex for students during implementation. Here, we discuss its importance in developing and delivering an educational system in Bioinformatics based on e-learning environment.
\end{abstract}

Keywords: e-learning; virtual learning

\section{Background:}

Bioinformatics has become a frontline applied science and it is of vital importance to the study of biology. This is widely recognized as the defining scientific endeavor of the twenty first century and this sector attains levels demanded in the international arena. This achievement can be through organizational and functional restructuring; integration and optimal initialization of the available resources; increased autonomy of the system; sustainable development of human resources; and the development of teaching or elearning modules for Bioinformatics. Lack of adequate skilled professionals is a major bottleneck for bioinformatics initiatives in India. The current challenge is to develop e-learning modules that would result in the generation of trained work force in bioinformatics. In recent years, distribution of computers, their amplifying multimedia performance, as well as increased access to internet of high bandwidth [1] leads to a great curiosity in the application of new technologies in education. Initially computer based training (CBT) was promoted via floppy disks and later in multimedia version on CD-ROM and DVD. [2] However, in this modern era the foremost computer based training is by the means of internet, that is web-based training (WBT), at present mostly equivalent to E-learning. Advanced technologies in computer and internet have made the E-learning a new approach for teaching. Many new technologies exist for communicating between two individuals or groups. Now at the convergence of computing and telecommunications, the ability to store and transmit knowledge is available, making it possible to transmit vast amounts of information anywhere in the world at an ever-increasing cost. These new technologies greatly facilitate the acquisition and absorption of knowledge, offering opportunities to enhance educational systems. Recently a Bioinformatics group [3] has developed an e-learning environment named ISee [4] in Java platform, which delivers basic concepts of bioinformatics in GUI interface. The module lack in advanced concepts and has thus failed to implement new technologies to the learning environment. So, in this paper we discussed about developing and delivering an e-learning environment for bioinformatics with advanced technologies.

\section{Description:}

Bioinformatics is conceptualizing biology in terms of molecules (in the sense of physical chemistry) and applying informatics techniques, derived from disciplines such as applied mathematics, computer science and statistics, to understand and organize the information associated with the biological molecules. [5] Therefore, the content required for developing an e-learning module, analysis, design and implementation requires expert organization. [6] A Blueprint of the proposed e-learning module is illustrated (Figure 1).

\section{Content development (Phase I)}

(1) Data collection: Data required on relevant topics are collected. Data collection includes advanced topics; (2) Data processing: Subject matter specialist defines the objectives and analyzes the delivery method. Breaking of content into manageable chunks that is meaningful to the objective and designing data navigation; (3) Text incorporation: Condensing detailed text in to briefs, so that it is not more than 6 to 10 lines per screen without changing its original meaning. The indexing of complete text within module for quick reference is also a need.

\section{Instructional design (Phase II)}

Incorporating quizzes and tests:

Designing questions and puzzles at the end of each module. Graphical representation of puzzles and their solutions is a necessity.

\section{Multimedia and web design (Phase III)}

Multimedia incorporation:

(1) incorporation of audio and indexing the audio details; (2) designing of video clips followed by questions; (3) creating scenario for relevant topics; (4) defining animation graphic element; (5) animation using graphic elements already developed (the complex process and the concept that are difficult to understand are animated); (6) merging of sounds in animation; and (7) developing web interfaces using synchronous and asynchronous techniques. 


\section{Bioinformation}

Note: In synchronous technique, there is real time communication like video conference and live chat, while in asynchronous technique the interlocutor to choose answering the message at a later time, using e-mails, discussion forums, and blogs.

\section{E-Learning environment (Phase IV)} Testing and execution:

A survey generated by students is used to quality assure the module for clarity and accuracy. Each part of the module is aligned and linked with others for producing a single pack. The entire module is then converted to executables for a package.

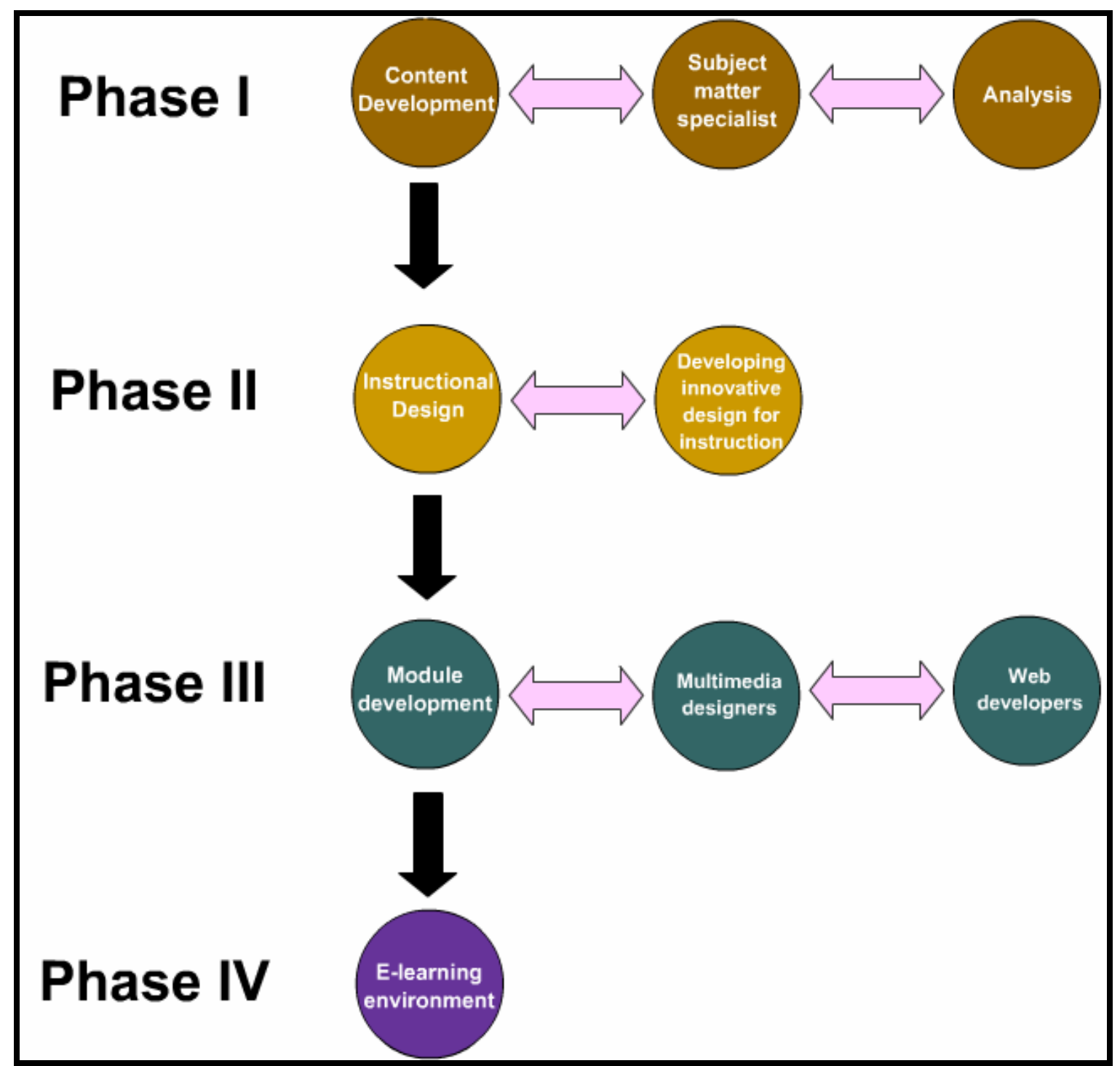

Figure 1: A blueprint of proposed E-learning module

\section{Conclusion:}

In this article, we described to create basic and advanced knowledge in bioinformatics for improved teaching. Bioinformatics is a fast growing discipline and therefore, the domain knowledge gathered in a discrete manner becomes obsolete within a short period. As such, there is an acute requirement for continuous training of students and researchers in emerging areas and concepts. [7] In view of this, the e-content creation is to provide continued education to the practicing bioinformaticians. It exploits interactive technologies and communication systems to improve learning experience and it has the potential to transform the way we teach and learn across the board. It can also rise standards and widen participation in life long learning. The E-learning method cannot replace lecturers and teachers, but it can enhance the quality of teaching and reduce the time spent on administration. It can enable every learner to achieve his or her potential and help to build an educational workforce empowered to change. It makes it possible for a truly ambitious education system for a future learning society. The challenge is in developing high quality e-content, as well as expertise for generating such content over long term. The e-content development and associated web learning does not seek to replace traditional teaching and learning, but is expected to supplement. The 


\section{Bioinformation}

by Biomedical Informatics Publishing Group

open access

\section{www.bioinformation.net}

\section{Views \& Challenges}

e-content learning is inevitable and hence it is important to design them to meet new challenges.

\section{References:}

[1] http://www.internetworldstats.com/stats.htm

[2] L. Whitfield, Health Serv J., 24: 117 (2007) [PMID: 17580767]

[3] http://www-helix.inrialpes.fr
[4] http://www-helix.inrialpes.fr/article124.html

[5] N. M. Luscombe, et al., Methods Inf Med., 40: 346 (2001) [PMID: 11552348]

[6] J. G. Ruiz, et al., Acad Med., 82: 503 (2007) [PMID: 17457075]

[7] A. Kowalewska, Rocz Panstw Zakl Hig., 57: 29 (2006) [PMID: 17472059]

Edited by R. Shanmugham

Citation: Saravanan \& Shanmughavel, Bioinformation 2(3): 83-85 (2007)

License statement: This is an open-access article, which permits unrestricted use, distribution, and reproduction in any medium, for non-commercial purposes, provided the original author and source are credited. 